\title{
FREE LIE ALGEBRAS AS MODULES FOR SYMMETRIC GROUPS
}

\author{
R. M. BRYANT, L. G. KOVÁCS and RALPH STÖHR \\ Dedicated to M. F. (Mike) Newman on the occasion of his 65th birthday
}

(Received 2 February 1999; revised 5 May 1999)

Communicated by C. F. Miller

\begin{abstract}
Let $r$ be a positive integer, $\mathbb{F}$ a field of odd prime characteristic $p$, and $L$ the free Lie algebra of rank $r$ over $\mathbb{F}$. Consider $L$ a module for the symmetric group $\mathcal{S}_{r}$ of all permutations of a free generating set of $L$. The homogeneous components $L^{n}$ of $L$ are finite dimensional submodules, and $L$ is their direct sum. For $p \leq r<2 p$, the main results of this paper identify the non-projective indecomposable direct summands of the $L^{n}$ as Specht modules or dual Specht modules corresponding to certain partitions. For the case $r=p$, the multiplicities of these indecomposables in the direct decompositions of the $L^{n}$ are also determined, as are the multiplicities of the projective indecomposables. (Corresponding results for $p=2$ have been obtained elsewhere.)
\end{abstract}

1991 Mathematics subject classification (Amer. Math. Soc.): primary 17B01; secondary 20C30.

\section{Introduction}

Let $r$ be an integer with $r \geq 2$ and $L$ a free Lie algebra over a field $\mathbb{F}$, freely generated by $r$ elements. Each permutation of these free generators extends uniquely to an automorphism of $L$, and so $L$ becomes a module for the symmetric group $\mathfrak{S}_{\text {r }}$ of degree $r$. For each positive integer $n$, let $L^{n}$ denote the homogeneous component of degree $n$ in $L$. Each $L^{n}$ is an $\mathbb{F S}_{r}$-submodule, and $L$ is the direct sum of the $L^{n}$, so the investigation of the module structure of $L$ reduces to that of the $L^{n}$. In particular, each indecomposable direct summand of $L$ is isomorphic to a direct summand of some

The authors gratefully acknowledge support from the Volkswagen-Stiftung (RiP-program at Oberwolfach) and the EPSRC.

(C) 1999 Australian Mathematical Society 0263-6115/99 $\$$ A2.00+0.00 
$L^{n}$. When the characteristic of $\mathbb{F}$ is 0 , the character $\chi_{n}$ of $\mathfrak{S}_{r}$ afforded by $L^{n}$ can be obtained from a formula due to Brandt [3]: for each $x$ in $\mathfrak{S}_{r}$,

$$
\chi_{n}(x)=\frac{1}{n} \sum_{d \mid n} \mu(d) \chi_{1}\left(x^{d}\right)^{n / d}
$$

where $\mu$ is the Möbius function. Using this, the multiplicities of the simple modules in $L^{n}$ may be determined by a straightforward application of the orthogonality relations. If the characteristic is a prime $p$, the same formula yields the relevant Brauer character, and orthogonality relations enable one to identify the composition factors and calculate their multiplicities. When $r<p$, the modules are semisimple so this information is decisive, but in general the $L^{n}$ cannot be expected to be semisimple. The aim of this paper is to resolve the difficulties which arise from this in the easiest cases, when $p \leq r<2 p$. For $p=2$, the matter has been settled in [4] and [8], so to avoid having to deal with exceptions we assume $p>2$.

Perhaps the most important modules for $\mathfrak{S}_{r}$ are the Specht modules $S^{\lambda}$, one for each partition $\lambda$ of $r$. Recall that these may be defined over $\mathbb{Z}$, and can then be interpreted over any other commutative ring with 1 by the usual 'change of scalars'. Over a field of characteristic 0 , they form a complete set of representatives of the isomorphism classes of simple $\mathfrak{S}_{r}$-modules. When working over a field of characteristic $p$, one focuses on $p$-regular partitions: partitions which do not have $p$ (or more) equal parts. (Among the partitions of $p$, only $\left(1^{p}\right)$ fails to be $p$-regular.) Each Specht module corresponding to a $p$-regular partition of $r$ has a unique maximal submodule, and the simple quotients form a complete set of representatives of the isomorphism classes of simple $\mathfrak{S}_{r}$-modules. Consequently, the projective covers of these Specht modules form a complete set of representatives of the isomorphism classes of projective indecomposable $\mathfrak{S}_{r}$-modules. Over any field, all simple $\mathfrak{S}_{r}$-modules are self-dual and absolutely simple. For notation and details, see James [7], particularly Theorem 11.5.

Our first result concerns the case $r=p$.

THEOREM 1. Let $p$ be an odd prime, $\mathbb{F}$ a field of characteristic $p$, and $L$ a free Lie algebra of rank $p$ over $\mathbb{F}$, viewed as an $\mathbb{F S}_{p}$-module where $\mathfrak{S}_{p}$ acts by permuting a free generating set of $L$. Then each non-projective indecomposable direct summand of $L$ is isomorphic to a Specht module $S^{\lambda}$ or its dual, where $\lambda$ is a partition of the form $\left(p-k, 1^{k}\right)$ with $k$ odd and $1 \leq k \leq p-2$.

In other words, $L$ is a direct sum of copies of projective indecomposable modules, Specht modules and dual Specht modules (corresponding to partitions of the kind indicated). This is a special case of the following somewhat more general result.

THEOREM 2. Let $p$ be an odd prime, $r$ an integer such that $p \leq r<2 p$, F a field of characteristic $p$, and $L$ a free Lie algebra of rank $r$ over $\mathbb{F}$, viewed as an $\mathbb{F S}_{r}$-module 
where $\mathfrak{S}_{r}$ acts by permuting a free generating set of $L$. Then each non-projective simple direct summand of $L$ is isomorphic to a Specht module $S^{\lambda}$ corresponding to a partition $\lambda$ of $r$ such that $\lambda_{1}>p$ and $\lambda_{1} \geq \lambda_{2}+p$, while each non-projective non-simple indecomposable direct summand is isomorphic either to an $S^{\lambda}$ such that $\lambda$ contains a p-hook of odd leg-length or to the dual of such an $S^{\lambda}$.

We prove Theorem 1 and Theorem 2 in Section 4 and Section 5, respectively. In the case of Theorem 1 we also completely settle the question of Krull-Schmidt multiplicities: we give a method for calculating, for each $n$, the multiplicity of each indecomposable (projective or not) in an unrefinable direct decomposition of $L^{n}$. Our result is Theorem 4, stated and proved in the second half of Section 4.

The arguments we use are much more general than required for the results already stated. To describe these arguments, we need some notation.

Given a field $\mathbb{E}$, a group $G$ and an $\mathbb{F} G$-module $V$, let $L(V)$ be the free Lie algebra over $\mathbb{F}$ of rank equal to the (possibly infinite) dimension of $V$, identify the first homogeneous component with $V$, and extend the action of $G$ on $V$ to an action on $L(V)$ by Lie algebra automorphisms. The homogeneous components $L^{n}(V)$ of $L(V)$ are then also $\mathbb{F} G$-modules, known as the Lie powers of $V$.

Now suppose that $\mathbb{F}$ has prime characteristic $p$ (not necessarily odd) while $G$ is finite and a Sylow $p$-subgroup $P$ of $G$ has order $p$ and is its own centralizer in $G$. Suppose also that $V$ is finite dimensional and projective. (These assumptions hold in Theorem 1 with $G=\mathfrak{S}_{p}$ and $V=L^{1}$.) [5, Theorem 1] yields that all non-projective indecomposable direct summands of $L^{n}(V)$ as $\mathbb{F} P$-module have dimension $p-1$. We use the Green correspondence to derive from this, in Lemma 2 of Section 2, that the non-projective indecomposable direct summands of $L^{n}(V)$ as $\mathbb{F} G$-module are odd Heller translates $\Omega^{2 i-1} \mathbb{F}_{G}$ of the 1-dimensional trivial $\mathbb{F} G$-module $\mathbb{F}_{G}$. Exploiting the methods of [5] further, in Theorem 3 of Section 3 we show how to obtain the KrullSchmidt multiplicities of all indecomposables in $L^{n}(V)$. In the case where $G=\mathfrak{S}_{p}$, the relevant Heller translates may be recognized as Specht modules or dual Specht modules.

When $G=\mathfrak{S}_{r}$ with $p<r<2 p, L^{1}$ is no longer a projective module. If $p+1<r<2 p$, then the Sylow subgroup $P$, while still of order $p$, is not its own centralizer. Nevertheless, the proof of Theorem 1 can be readily adapted to yield Theorem 2 as well.

\section{Groups with a self-centralizing Sylow subgroup of prime order}

In this section and in the next one, we shall not need the assumption that $p>2$.

Let $G$ be any finite group with a self-centralizing Sylow subgroup $P$ of prime order $p$. The representation theory of such a group over the field of $p$ elements (and 
over the ring of $p$-adic integers) has been elaborated in the last two sections of the lecture notes [6] of Green. Except for using $g, h$ and $j$ where [6] uses $\delta, \sigma$ and $\alpha$, respectively, we keep to the notation of [6] as we recall what we need here. What we do need does not depend on keeping to the prime field: $\mathbb{F}$ can be any field of characteristic $p$.

Let $N$ be the normalizer of $P$ in $G$; set $e=|N: P|$, and let $g$ be a generator of $P$. There exist cyclic complements to $P$ in $N$ : let $h$ be a generator for one of them, and let $l$ be the smallest positive integer such that $h^{-1} g h=g^{l}$. We shall also write $l$ for the coset $l+p \mathbb{Z}$ regarded as an element of $\mathbb{F}$ (in this sense, $l$ is a primitive $e$ th root of 1 ). Each indecomposable $\mathbb{F} N$-module is uniserial, even as $\mathbb{F} P$-module. Representatives of the isomorphism classes of the indecomposable $\mathbb{F} N$-modules may be labelled as $T_{i, j}$ with $1 \leq i \leq e$ and $1 \leq j \leq p$ in such a way that $\operatorname{dim} T_{i, j}=j$ and, on the top composition factor of this module, $h$ acts as multiplication by $l^{i}$ (and of course $g$ acts trivially). In particular, the simple $\mathbb{F} N$-modules are the $T_{i, 1}$, with $T_{e, 1}$ being the trivial simple module which is also denoted by $\mathbb{F}_{N}$, while the projective indecomposables are the $T_{i, p}$. If $j>1$, the unique maximal submodule of $T_{i, j}$ is $T_{i+1, j-1}$ (with $i+1 \mathrm{read}$ modulo $e$ : a similar convention applies throughout). From this, it is easy to see that each eigenspace of $h$ in $T_{i, p-1}$ has dimension $(p-1) / e$, while in $T_{i, p}$ the dimension of the eigenspace with eigenvalue $l^{i}$ is $1+(p-1) / e$ and all other eigenspaces have dimension $(p-1) / e$.

LEMMA 1. If an $\mathbb{F} N$-module $U$ of dimension $j$ can be generated by an eigenvector for $h$ with eigenvalue $l^{i}$, then $1 \leq j \leq p$ and $U \cong T_{i, j}$.

PROOF. Each element of $N$ is the product of a power of $h$ and an element of $P$ : therefore the $N$-orbit of an eigenvector for $h$ spans the same subspace as its $P$-orbit. Since the regular $\mathbb{F} P$-module is uniserial, so is every $\mathbb{F} P$-module generated by a single element, and therefore $U$ is uniserial. The generating eigenvector has non-zero image in the top composition factor of $U$, and so the claim follows.

By $[6,18.3]$, the odd Heller translates of $\mathbb{F}_{N}$ range through the $(p-1)$-dimensional indecomposables and form a periodic sequence with period $e$. Specifically, $T_{i, p-1} \cong \Omega^{2 i-1} \mathbb{F}_{N}$ (not $\Omega^{2 i+1} \mathbb{F}_{N}$ : that was a typographical error in the last line of 18.3). The Green correspondence (the inverse pair of the operators called $f$ and $g$ in [6]) commutes with the Heller operator $\Omega$ (see $[6,17.6 \mathrm{~b}]$ ), and the Green correspondent of the trivial simple $\mathbb{F} G$-module $\mathbb{F}_{G}$ can only be $\mathbb{F}_{N}$; therefore the Green correspondents of the $T_{i, p-1}$ are the odd Heller translates of $\mathbb{F}_{G}$. Being Heller translates of a nonprojective indecomposable, the latter are also non-projective indecomposables (see $[6,17.5 \mathrm{~b}])$. By the definition of Green correspondence, this yields the following.

LEMMA 2. If $U$ is a finite dimensional $\mathbb{F} G$-module, then the multiplicity of $\Omega^{2 i-1} \mathbb{F}_{G}$ 
in $U$ (with $1 \leq i \leq e$ ) is the same as the multiplicity of $T_{i, p-1}$ in the restriction $U \downarrow_{N}$. If all non-projective indecomposable direct summands of $U \downarrow_{p}$ have dimension $p-1$, then each non-projective indecomposable direct summand of $U$ is an $\Omega^{2 i-1} \mathbb{F}_{G}$.

\section{Non-projective indecomposables in Lie powers of projectives}

We continue with the assumptions and notation of the previous section. We are interested in the structure of the Lie powers $L^{n}(V)$, where $V$ is a finite dimensional projective $\mathbb{F} G$-module. In this case the restriction of $V$ to $P$ is free so that the restriction of $L^{n}(V)$ to $P$ is a Lie power of a free $\mathbb{F} P$-module.

In a recent paper [5], the first and last authors determined the $\mathbb{F} P$-module structure of such Lie powers. They proved that the indecomposable direct summands fall into two isomorphism types, one of dimension $p$ and the other of dimension $p-1$, and gave formulas for the relevant multiplicities. The key to those formulas was that if in the $n$th Lie power the multiplicity of the $(p-1)$-dimensional indecomposable is denoted by $\alpha(n)$ and the multiplicity of the $p$-dimensional (regular) indecomposable is denoted by $\rho(n)$, then $\alpha(n)$ is the sum of the $\rho(n / d)$ with $d$ ranging over the powers of $p$ that divide $n$ and are different from 1. With the dimension of the Lie power available from Witt's formula, once $\alpha(n)$ is known, so is $\rho(n)$. The link between $(p-1)$-dimensional direct summands in one Lie power and $p$-dimensional direct summands in smaller Lie powers was made quite explicit in [5], matching generators of one to generators of the other. The way this was done was designed to facilitate the extension of the result to projective modules for $\mathbb{F} N$, though the extension was not fully elaborated there. A more detailed analysis will take up most of the present section.

In [5], the group $N$ was defined somewhat differently, and only for $e=p-1$. But it is easy to see that the arguments of [5] work equally well for $N$ as defined here (with arbitrary $e$ ). Thus we shall interpret the results of [5] as applying to the situation here.

Let $U$ be a finite dimensional projective $\mathbb{F} N$-module. Then the restriction $L^{1}(U) \downarrow_{P}$ is free, so by [5, Theorem 1] all indecomposable direct summands of $L^{n}(U) \downarrow_{P}$ have dimension at least $p-1$. In terms of the previous section, it follows that we may write

$$
\mathrm{L}^{n}(U) \cong \bigoplus_{i=1}^{e} T_{i, p-1}^{\oplus \alpha(n, i)} \oplus \bigoplus_{i=1}^{e} T_{i, p}^{\oplus \rho(n, i)},
$$

so that $\alpha(n)=\sum_{i} \alpha(n, i)$ and $\rho(n)=\sum_{i} \rho(n, i)$. By assumption, all the $\alpha(1, i)$ vanish, and the $\rho(1, i)$ describe the structure of $U$. The next task is to calculate the $\alpha(n, i)$ and the $\rho(n, i)$ for $n>1$.

It is easy to see that each $T_{i, p}$ has a basis which is a $P$-orbit of an eigenvector of $h$, so $U$ has a basis which is the union of such orbits. This is exactly what is needed 
to make the results of [5] applicable here. Let $\mathrm{A}(U)$ denote the universal enveloping algebra of $\mathrm{L}(U)$. The action of $N$ on $U$ extends not only to an action on $\mathrm{L}(U)$ but also to an action on $\mathrm{A}(U)$. We shall make use of a map $\phi$ (not a homomorphism) of $\mathrm{A}(U)$ into itself which, like $p$ th powering, takes each eigenspace of $h$ into itself. Let us defer the definition of $\phi$ and the proof of this property until later in this section: for the moment we need to know nothing more about $\phi$. Recall that $g$ is a generator of $P$. By Theorem 6.4 and Proposition 6.5 of [5], there exist subsets $\mathscr{L}_{1}, \mathscr{L}_{2}, \ldots$ of $L(U)$ which consist of eigenvectors of $h$ and are such that, for each positive $n$, the union of

$$
\left\{u g^{k} \mid u \in \mathscr{L}_{n}, k=0,1, \ldots, p-1\right\}
$$

and

$$
\bigcup_{\beta \geq 1, p^{\beta} \mid n}\left\{\left(u^{p^{\beta-1}} \phi\right)\left(1-g^{k}\right) \mid u \in \mathscr{L}_{n / p^{\beta}}, k=1, \ldots, p-1\right\}
$$

is a basis of $L^{n}(U)$. (The union in the last display is, in effect, over the $p$-power divisors $p^{\beta}$ of $n$ which are greater than 1. It is not obvious, but proved in [5], that the $\left(u^{p^{\beta-1}}\right) \phi\left(1-g^{k}\right)$ lie in $\mathrm{L}(U)$ even when $\beta>1$.)

By Lemma 1 and the linear independence of the elements of the union, the span of any one $\left\{u g^{k} \mid k=0,1, \ldots, p-1\right\}$ is an indecomposable $\mathbb{F} N$-module, isomorphic to $T_{i, p}$, where $i$ satisfies $u h=l^{i} u$. Given any $u$ in an $\mathscr{L}_{n / p^{\beta}}$, the span of $\left\{\left(u^{p^{\beta-1}} \phi\right) g^{k}\right\}$ $k=0, \ldots, p-1\}$ is an $\mathbb{F} N$-module (being a submodule of A $(U)$ ). By Lemma 1, this module is indecomposable. It has the span of $\left\{\left(u^{p^{\beta-1}} \phi\right)\left(1-g^{k}\right) \mid k=1, \ldots, p-1\right\}$ as a submodule of dimension $p-1$. Thus it has dimension $p$ and is isomorphic to $T_{i, p}$, where $i$ satisfies $u h=l^{i} u$. It follows that the span of $\left\{\left(u^{p^{\beta-1}} \phi\right)\left(1-g^{k}\right) \mid k=\right.$ $1, \ldots, p-1\}$ is isomorphic to $T_{i+1, p-1}$. Our $L^{n}(U)$ is thus the direct sum of the $T_{i, p}$ corresponding to the $u$ in $\mathscr{L}_{n}$, and of the $T_{i+1, p-1}$ corresponding to the $u$ in the $\mathscr{L}_{n / p^{\beta}}$. This proves that precisely $\rho(n, i)$ elements of $\mathscr{L}_{n}$ correspond to the eigenvalue $l^{i}$, and that

$$
\alpha(n, i)=\sum_{\beta \geq 1, p^{\beta} \mid n} \rho\left(n / p^{\beta}, i-1\right),
$$

where $i-1$ is read modulo $e$. (In terms of the usual convention that the sum of an empty set of summands is 0 , this formula is consistent with all $\alpha(1, i)$ being 0 .)

Let $\theta(n, i)$ denote the dimension of the eigenspace of $h$ in $L^{n}(U)$ corresponding to the eigenvalue $l^{i}$. Using the eigenspace dimensions in the summands of the decomposition (3.1), we get that

$$
\theta(n, i)=\rho(n, i)+\frac{p-1}{e}(\alpha(n)+\rho(n))
$$


for $i=1, \ldots, e$. On the other hand, $\alpha(n)+\rho(n)$ is the dimension of the fixed point space of $g$ in $L^{n}(U)$, and that has been determined in [5, Corollary 1]. Hence

$$
\rho(n, i)=\theta(n, i)-\frac{p-1}{e p n} \sum_{d \mid n, p \nmid d} \mu(d)(\operatorname{dim} U)^{n / d} .
$$

(As before, $\mu$ stands for the Möbius function.)

In view of (3.2), this has reduced matters to calculating the eigenspace dimensions $\theta(n, i)$. Of course,

$$
\theta(1, i)=\rho(1, i)+\frac{p-1}{e p} \operatorname{dim} U .
$$

Given a free Lie algebra on free generators $x_{1,1}, \ldots, x_{1, r_{1}}, x_{2,1}, \ldots, x_{2, r_{2}}, \ldots$ and a sequence of non-negative integers $m_{1}, m_{2}, \ldots$ with finite, non-zero sum $\sum m_{j}$, let the dimension of the space spanned by the Lie monomials with $m_{1}$ entries from $\left\{x_{1,1}, \ldots, x_{1, r_{1}}\right\}, m_{2}$ entries from $\left\{x_{2,1}, \ldots, x_{2, r_{2}}\right\}, \ldots$ be denoted by $\kappa\left(r_{1}, r_{2}, \ldots ; m_{1}\right.$, $\left.m_{2}, \ldots\right)$. It was shown in Witt $[11$, Satz 6 and (7) page 201] that

$$
\kappa\left(r_{1}, r_{2}, \ldots ; m_{1}, m_{2}, \ldots\right)=\frac{1}{\sum m_{j}} \sum_{d} \mu(d) \frac{\left(\sum m_{j} / d\right) !}{\prod\left(m_{j} / d\right) !} \prod r_{j}^{m_{j} / d}
$$

with summation over all common divisors $d$ of $m_{1}, m_{2}, \ldots$ Apply this with $\left\{x_{j, 1}, \ldots\right.$, $\left.x_{j, r_{j}}\right\}$ a basis for the eigenspace of $h$ in $U$ with eigenvalue $l^{j}$, for $j=1,2, \ldots, e$. Then $r_{j}=\theta(1, j)$, and the subspace whose dimension we have denoted by $\kappa\left(r_{1}, \ldots, r_{e}\right.$; $\left.m_{1}, \ldots, m_{e}\right)$ is part of the eigenspace with eigenvalue $l^{m}$ where $m=\sum_{j=1}^{e} j m_{j}$. It follows that

$$
\theta(n, i)=\sum \kappa\left(\theta(1,1), \ldots, \theta(1, e) ; m_{1}, \ldots, m_{e}\right)
$$

with summation over all sequences $m_{1}, \ldots, m_{e}$ of non-negative integers such that $\sum m_{j}=n$ and $\sum j m_{j} \equiv i(\bmod e)$.

This completes the description of our method for calculating the multiplicities $\alpha(n, i)$ and $\rho(n, i)$.

It is time to give the deferred definition of the map $\phi$ and establish the only property of it that we have used. To this end, let $A$ denote the free associative ring on free generators $x_{0}, x_{1}, \ldots, x_{p-1}$ and take the usual Lie bracket operation defined by $[a, b]=a b-b a$ for all $a, b$ in $A$. Consider the element $w$ of $A$ given by

$$
w=\frac{1}{p}\left(\sum_{\pi} x_{\pi(0)} \cdots x_{\pi(p-1)}-\sum_{\sigma}\left[x_{0}, x_{\sigma(1)}, \ldots, x_{\sigma(p-1)}\right]\right),
$$

where $\pi$ runs over all permutations of $\{0,1, \ldots, p-1\}, \sigma$ runs over all permutations of $\{1, \ldots, p-1\}$, and the terms in the second sum are left-normed Lie products. (At 
first sight, division by $p$ does not make sense here, but it was shown by Wall [10] that the expression in parentheses belongs to $p A$.) Given an element $u$ in $\mathrm{A}(U)$, we define $u \phi$ as the image of $w$ under the ring homomorphism $\phi_{u}$ which, for $j=0, \ldots, p-1$, maps $x_{j}$ to $u g^{j}$.

Suppose now that $u$ is an eigenvector for $h$ in $\mathrm{A}(U)$, with eigenvalue $l^{i}$ : our last debt is to show that in this case $u \phi$ is also an eigenvector, with the same eigenvalue. Notice first that the element $w$ is symmetric in $x_{1}, \ldots, x_{p-1}$ (that is, invariant under any automorphism of $A$ that fixes $x_{0}$ and permutes the other free generators $x_{1}, \ldots, x_{p-1}$ ) and linear in each $x_{j}$ (that is, if an endomorphism of $A$ maps $x_{j}$ to a scalar multiple and fixes each of the other free generators, then it maps $w$ to its multiple by the same scalar). Given an integer $i$, consider the endomorphism $\varepsilon_{i}$ of $A$ defined by $x_{j} \varepsilon_{i}=l^{i} x_{l j}$ for $j=0,1, \ldots, p-1$ where the subscript $l j$ is read modulo $p$ : by the preceding observations, $w \varepsilon_{i}=l^{i p} w$. Here $l^{i}$ was regarded as an element of $\mathbb{Z}$; using also its interpretation in $u h=l^{i} u$ as an element of $\mathbb{F}$, one can argue that $\left(x_{j} \phi_{u}\right) h=\left(u g^{j}\right) h=(u h) g^{l j}=l^{i} u g^{l j}=\left(x_{j} \varepsilon_{i}\right) \phi_{u}$. As $\left(x_{j} \phi_{u}\right) h=\left(x_{j} \varepsilon_{i}\right) \phi_{u}$ holds for each of the generators $x_{j}$, it holds for every element of $A$ in place of $x_{j}$. In particular, it holds for $w$, and so $(u \phi) h=\left(w \phi_{u}\right) h=\left(w \varepsilon_{i}\right) \phi_{u}=\left(l^{i p} w\right) \phi_{u}=l^{i}(u \phi)$, as required.

We have established the following result.

LEMMA 3. Let $U$ be any finite dimensional projective $\mathbb{F} N$-module, written as $U=$ $\bigoplus_{i=1}^{e} T_{i, p}^{\oplus \rho(1, i)}$. The indecomposable direct summands of the Lie power $L^{n}(U)$ of $U$ are then the $T_{i, p}$ and the $T_{i, p-1}$, as in (3.1); the multiplicities $\rho(n, i)$ are given by (3.3) (which relies on (3.6), (3.4), (3.5), in turn), and then the $\alpha(n, i)$ are given by (3.2).

In view of Lemma 2 , this proves our main technical result.

THEOREM 3. Let $p$ be any prime, $\mathbb{F}$ any field of characteristic $p$, and $G$ a finite group in which a Sylow p-subgroup is self-centralizing and of order p. Let $e$ be the index of that Sylow subgroup in its normalizer $N$. If $V$ is a finite dimensional projective $\mathbb{F} G$-module, then the non-projective indecomposable direct summands of the Lie powers of $V$ are Heller translates $\Omega^{2 i-1} \mathbb{F}_{G}$ of the 1-dimensional trivial $\mathbb{F} G$ module, with $1 \leq i \leq e$. Using the notation of the previous section, write the restriction of $V$ to $N$ as $V \downarrow_{N}=\bigoplus_{i=1}^{e} T_{i, p}^{\oplus \rho(1, i)}$ and let the numbers $\alpha(n, i)$ be as given by Lemma 3. Then the multiplicity of $\Omega^{2 i-1} \mathbb{F}_{G}$ in $\mathrm{L}^{n}(V)$ is precisely $\alpha(n, i)$.

There is much more about representations of such groups $G$ in Green's notes [6] that could be used in this generality. Nevertheless, we now turn to the special case of central interest here, to $G=\mathfrak{S}_{p}$.

\section{Symmetric groups of degree $p$}

To avoid exceptions, from now on we assume that $p$ is odd. 
Consider representations over an arbitrary field $\mathbb{F}$ of characteristic $p$. As in James [7], if $\lambda$ is a $p$-regular partition, the unique simple quotient of $S^{\lambda}$ will be written as $D^{\lambda}$. These $D^{\lambda}$ are pairwise non-isomorphic.

The only partition of $p$ that is not $p$-regular is $\left(1^{p}\right)$. It has been known at least since Nakayama [9] that if $\lambda$ is a partition of $p$ that is not a hook partition (that is, if more than one part of $\lambda$ is greater than 1), then $S^{\lambda}$ is simple and projective. The remaining part of the decomposition matrix for $\mathbb{F S}_{p}$ is easily deduced from [9, Theorem 4] (or looked up in [7, 24.1]). Of course, $S^{(p)}$ and $S^{\left(1^{p}\right)}$ afford the 1-dimensional trivial and alternating representations of $\mathfrak{S}_{p}$, respectively. (Note that, as $\left(1^{p}\right)$ is not a $p$-regular partition, the preferred $p$-modular recognition of the alternating representation of $\mathfrak{S}_{p}$ is not as $S^{\left(1^{p}\right)}$ but as $D^{\left(2,1^{p-2)}\right)}$.) The Specht modules corresponding to the other hook partitions of $p$ are uniserials of composition length 2 , the unique simple submodule of $S^{\left(p-k, 1^{k}\right)}$ being isomorphic to $D^{\left(p-k+1,1^{k-1}\right)}$ whenever $1 \leq k \leq p-2$. These Specht modules are non-projective, because their dimensions are prime to $p$ : by the hook length formula, 20.1 in [7], we have $\operatorname{dim} S^{\left(p-k, 1^{k}\right)}=\left(\begin{array}{c}p-1 \\ k\end{array}\right)$.

For each $p$-regular partition $\lambda$ of $p$, let $p^{\lambda}$ denote the projective cover of $S^{\lambda}$. If $\lambda$ is not a hook partition, then $P^{\lambda}=S^{\lambda}$. Now suppose $\lambda$ is a hook partition. Then $\Omega S^{\lambda}$ is a submodule of $P^{\lambda}$ with quotient $S^{\lambda}$, and the top and bottom composition factors of $P^{\lambda}$ are isomorphic to $D^{\lambda}$, so we know that $\Omega S^{\lambda}$ has a unique simple submodule and that is isomorphic to $D^{\lambda}$. The decomposition matrix has, in effect, been described above. Since all fields are splitting fields for the symmetric groups, the Cartan matrix is the product of the decomposition matrix and its transpose (in the opposite order). It follows that the heart of $P^{(p)}$ (that is, the quotient of the unique maximal submodule over the unique simple submodule) is a $D^{(p-1,1)}$, the heart of $P^{\left(2,1^{p-2}\right)}$ is a $D^{\left(3,1^{p-3}\right)}$, while if $1 \leq k \leq p-3$ then the heart of $P^{\left(p-k, 1^{k}\right)}$ has two non-isomorphic composition factors, a $D^{\left(p-k+1,1^{k-1}\right)}$ and a $D^{\left(p-k-1,1^{k+1}\right)}$. It can be seen from this that $P^{\lambda}$ has only one submodule whose quotient has the same composition factors as $S^{\lambda}$. Therefore, if a uniserial module has the same composition factors, in the same order, as $S^{\lambda}$, then it is isomorphic to $S^{\lambda}$. One can also read off the composition factors of the $\Omega S^{\lambda}$, and the orders of these factors, and so conclude that $\Omega S^{\left(p-k, 1^{k}\right)} \cong S^{\left(p-k-1,1^{k+1}\right)}$ whenever $0 \leq k \leq p-3$, while $\Omega S^{\left(2,1^{p-2}\right)} \cong D^{\left(2,1^{p-2}\right)}$. This proves that

$$
\Omega^{k} \mathbb{F}_{\mathfrak{G}_{p}} \cong S^{\left(p-k, 1^{k}\right)} \quad \text { when } 1 \leq k \leq p-2,
$$

while

$$
\Omega^{p-1} \mathbb{F}_{\mathfrak{S}_{p}} \cong D^{\left(2,1^{p-2}\right)}
$$

To prove Theorem 1, it remains to exploit Heller's Lemma (see Green $[6,17.5 \mathrm{c}]$ ). For a non-projective indecomposable $V$, this says that $\Omega\left(V^{*}\right)=\left(\Omega^{-1}(V)\right)^{*}$. As $D^{\left(2, p^{p-2}\right)}$ (like every simple module for a symmetric group) is self-dual, it follows that 
$\Omega D^{\left(2,1^{p-2}\right)} \cong\left(S^{\left(2,1^{p-2}\right)}\right)^{*}$ while $\Omega\left(\left(S^{\left(p-k-1,1^{k+1}\right)}\right)^{*}\right) \cong\left(S^{\left(p-k, 1^{k}\right)}\right)^{*}$ whenever $0 \leq k \leq$ $p-3$. Thus

$$
\Omega^{k} \mathbb{F}_{\mathfrak{S}_{p}} \cong\left(S^{\left(k+2-p, 1^{2 p-2-k}\right)}\right)^{*} \text { when } p \leq k \leq 2 p-2
$$

Hence

$$
\Omega^{2 i-1} \mathbb{F}_{\mathcal{S}_{p}} \cong \begin{cases}S^{\left(p+1-2 i, 1^{2 i-1}\right)} & \text { if } 1 \leq i \leq(p-1) / 2, \\ \left(S^{\left(2 i+1-p, 1^{2 p-1-2 i}\right)}\right)^{*} & \text { if }(p+1) / 2 \leq i \leq p-1 .\end{cases}
$$

(Note that the Specht modules whose duals occur in the second line are the same as the Specht modules in the first line, just listed in the opposite order.) In view of the first part of Theorem 3, this proves Theorem 1.

We conclude this section by describing how the Krull-Schmidt multiplicities in $L^{n}$ may be calculated under the assumptions of Theorem 1 .

For each partition $\lambda$ of $p$, let $\nu(n, \lambda)$ denote the multiplicity of the Specht module $S^{\lambda}$ in the homogeneous component of degree $n$ in the free Lie algebra of rank $p$ over the rational field $\mathbb{Q}$. The first step is to calculate these numbers (from Brandt's formula and the orthogonality relations, or otherwise). The $v(n, \lambda)$ corresponding to hook partitions will enter into our formulas as alternating sums, so it will be convenient to define, for $k=0, \ldots, p-2$,

$$
v_{n}(k)=v\left(n,\left(p-k, 1^{k}\right)\right)-v\left(n,\left(p-k+1,1^{k-1}\right)\right)+\cdots \pm v(n,(p)) .
$$

Next, in Lemma 3 of Section 3, set the initial conditions as $\rho(1, i)=0$ for $1 \leq i \leq$ $p-2$ and $\rho(1, p-1)=1$, in order to obtain the $\alpha(n, i)$. Fix this as the meaning of $\alpha(n, i)$ for the sequel. In terms of these numbers, let

$$
\pi_{n}(p-2)=\frac{1}{p}\left((p-1) v\left(n,\left(1^{p}\right)\right)+v_{n}(p-2)-\sum_{i=1}^{p-1} \alpha(n, i)\right)
$$

and, for $k=0, \ldots, p-3$,

$$
\begin{aligned}
\pi_{n}(k)= & v_{n}(k)+(-1)^{k}(k+1)\left(\pi_{n}(p-2)-v\left(n,\left(1^{p}\right)\right)\right) \\
& +(-1)^{k} \sum(\alpha(n, i)+\alpha(n, p-i))
\end{aligned}
$$

where summation is over $i$ ranging from 1 to $(k+1) / 2$ (rounded down) and at $k=0$ the sum with no summands is interpreted as 0 .

THEOREM 4. Let $L$ be as in Theorem 1 and consider a homogeneous component $L^{n}$ of $L$. For odd $k$ with $1 \leq k \leq p-2$, the multiplicity of $S^{\left(p-k, \mathrm{I}^{k}\right)}$ in $L^{n}$ is $\alpha(n,(k+1) / 2)$, and the multiplicity of the dual of $S^{\left(p-k, 1^{k}\right)}$ is $\alpha(n, p-(k+1) / 2)$. For $0 \leq k \leq p-2$, the multiplicity of the projective cover of $S^{\left(p-k .1^{k}\right)}$ in $L^{n}$ is $\pi_{n}(k)$. If $\lambda$ is a partition of $p$ that is not a hook partition, then the multiplicity of $S^{\lambda}$ in $L^{n}$ is $v(n, \lambda)$. 
PROOF. Theorem 3 and (4.1) justify the claim concerning the multiplicities of the Specht modules and dual Specht modules corresponding to hook partitions.

For each partition $\lambda$ of $p$, let $\chi^{\lambda}$ denote the (ordinary) character of $\mathfrak{S}_{p}$ afforded by the Specht module $S^{\lambda}$ defined over $\mathbb{Q}$. Note that these characters take their values in $\mathbb{Z}$. By the definition of the multiplicities $v$, the character $\chi_{n}$ of $\mathfrak{S}_{p}$ afforded by the homogeneous component of the free Lie algebra of rank $p$ over $\mathbb{Q}$ may be written as $\chi_{n}=\sum v(n, \lambda) \chi^{\lambda}$ with summation over all partitions $\lambda$ of $p$. Restriction to $p^{\prime}$ elements converts $\chi_{n}$ into the Brauer character of our $L^{n}$, and the $\chi^{\lambda}$ into the Brauer characters of the Specht modules defined over $\mathbb{F}$. As all simple $\mathfrak{S}_{p}$-modules are self-dual, here the Brauer character of the dual of a module is always the same as the Brauer character of the original module. It follows that the Brauer character of the largest projective direct summand of $L^{n}$ is the restriction of

$$
\sum_{\lambda} \nu(n, \lambda) \chi^{\lambda}-\sum_{i=1}^{(p-1) / 2}(\alpha(n, i)+\alpha(n, p-i)) \chi^{\left(p+1-2 i, 1^{2 i-1}\right)} .
$$

The restriction of $\chi^{\left(1^{p}\right)}$ is the negative of the restriction of $\sum_{k=0}^{p-2}(-1)^{k} \chi^{\left(p-k, 1^{k}\right)}$ (see James [7, first line of page 99]), so $\chi^{\left(1^{p}\right)}$ may be replaced in (4.2) by this alternating sum, yielding a linear combination of characters corresponding to $p$-regular partitions.

Given the decomposition matrix described above, Brauer reciprocity (Green [6, 13.5a]) gives that the Brauer character of $P^{\lambda}$ is the restriction of

$$
\begin{array}{ll}
\chi^{\left(p-k, 1^{k}\right)}+\chi^{\left(p-k-1,1^{k+1}\right)} & \text { if } \lambda=\left(p-k, 1^{k}\right) \text { with } 0 \leq k \leq p-2, \\
\chi^{\lambda} & \text { if } \lambda \text { is not a hook partition. }
\end{array}
$$

For each $p$-regular partition $\lambda$ of $p$, write $\pi_{\lambda}$ for the multiplicity of $P^{\lambda}$ in $L^{n}$ : then the Brauer character of the largest projective summand of $L^{n}$ is the restriction of

$$
\sum_{\text {non-hook } \lambda} \pi_{\lambda} \chi^{\lambda}+\sum_{k=0}^{p-2} \pi_{\left(p-k, 1^{k}\right)}\left(\chi^{\left(p-k, 1^{k}\right)}+\chi^{\left(p-k-1,1^{k+1}\right)}\right) .
$$

The restrictions of the $\chi^{\lambda}$ with $p$-regular $\lambda$ are linearly independent (use James [7, 12.3]), so-after eliminating $\chi^{\left(1^{p}\right)}$ from (4.2) and (4.3) as indicated-we can equate coefficients in the two resulting expressions for the one Brauer character. This gives a system of simultaneous linear equations for the $\pi_{\lambda}$, and the unique solution of that is readily seen to be

$$
\pi_{\lambda}= \begin{cases}\pi_{n}(k) & \text { if } \lambda=\left(p-k, 1^{k}\right) \text { with } 0 \leq k \leq p-2, \\ v(n, \lambda) & \text { if } \lambda \text { is not a hook partition. }\end{cases}
$$

The proof of Theorem 4 is now complete.

In view of Theorem 1, Theorem 4 accounts for all the Krull-Schmidt multiplicities in $L^{n}$. 


\section{Symmetric groups of degree between $p$ and $2 p$}

It will be convenient to keep to odd $p$. The aim of this section is to prove Theorem 2 .

Except as indicated, the argument has the same structure as the proof of Theorem 1, so we shall only sketch it. We have seen that all Specht modules mentioned in that theorem are non-simple; thus, for the case $r=p$, Theorem 2 follows immediately from Theorem 1. Suppose for the sequel that $p<r<2 p$. Note that no partition of so small an $r$ can have more than one $p$-hook. If a partition of $r$ is not $p$-regular, each of the last $p$ non-zero parts must be 1 , and these form a $p$-hook. In the terminology of James [7], the leg-length of this hook is $p-1$. When $\lambda$ is a partition of $r$ such that $\lambda_{1} \geq \lambda_{2}+p$, the unique $p$-hook has leg-length 0 . Thus all partitions mentioned in Theorem 2 are $p$-regular.

The normalizer of a Sylow $p$-subgroup in $\mathfrak{S}_{r}$ is now a direct product $R \times N$ where $R=\mathfrak{S}_{r-p}$ and $N$ is the Sylow normalizer in $\mathfrak{S}_{p}$, as before. The indecomposable $\mathbb{F}(R \times N)$-modules are the outer tensor products $S^{\mu} \sharp T_{i, j}$ where $\mu$ ranges through the partitions of $r-p$ and the $T_{i, j}$ are as defined in Section 2. The $S^{\mu} \sharp T_{i, p-1}$ are readily seen to be the odd Heller translates of the $S^{\mu} \sharp \mathbb{F}_{N}$.

We need one extra tool: the (Lazard) Elimination Theorem (Bourbaki [2, Chap. II, $\S 2.9$, Proposition 10]), which may be invoked here as follows. We have $L^{1} \downarrow_{R \times N} \cong$ $\left(U \sharp \mathbb{F}_{N}\right) \oplus\left(\mathbb{F}_{R} \sharp T_{p-1, p}\right)$ where $U$ is the natural $\mathbb{F} R$-module. The Elimination Theorem yields that

$$
L \downarrow_{R \times N} \cong\left(\mathrm{L}(U) \sharp \mathbb{F}_{N}\right) \oplus \mathrm{L}\left(\mathrm{T}(U) \sharp T_{p-1, p}\right)
$$

where $T(U)$ is the tensor algebra on $U$. The indecomposables in the first direct summand are all non-projective, of the form $S^{\mu} \sharp \mathbb{F}_{N}$. On the other hand, as $T_{p-1, p}$ is a projective $\mathbb{F} N$-module, the second direct summand as $\mathbb{F} N$-module is a free Lie algebra on a projective module. This projective module is infinite dimensional, but the qualitative part of Theorem 1 of [5] still applies (as one readily sees from [5, Theorem 2]): the non-projective $\mathbb{F} N$-indecomposables involved in $\mathrm{L}\left(\mathrm{T}(U) \sharp T_{p-1, p}\right)$ are all of dimension $p-1$, that is, they are odd Heller translates of $\mathbb{F}_{N}$. It follows that the non-projective indecomposable $\mathbb{F}(R \times N)$-modules involved in $\mathrm{L}\left(\mathrm{T}(U) \sharp T_{p-1, p}\right)$ are of the form $S^{\mu} \sharp\left(\Omega^{2 i-1} \mathbb{F}_{N}\right)$, that is, $\Omega^{2 i-1}\left(S^{\mu} \sharp \mathbb{F}_{N}\right)$. The Green correspondence then yields that the non-projective indecomposables involved in $L$ are the Green correspondents of the $S^{\mu} \sharp \mathbb{F}_{N}$ and the odd Heller translates of these modules. (For the Green correspondence in this generality, see for example Alperin [1, Section 10]. In that book the ground field is assumed algebraically closed, but this is not used in Section 10.)

Given any partition $\mu$ of $r-p$, let $\lambda$ be defined by setting $\lambda_{1}=\mu_{1}+p$ and $\lambda_{i}=\mu_{i}$ whenever $i>1$ : we claim that the Green correspondent of $S^{\mu} \sharp \mathbb{F}_{N}$ is $S^{\lambda}$. To see this, note first that $S^{\lambda}$ is indecomposable, because it is a Specht module corresponding 
to a $p$-regular partition. Next, the order of $R$ is prime to the characteristic $p$, so the indecomposable $\mathbb{F}\left(R \times \mathbb{S}_{p}\right)$-modules are the outer tensor products of the simple $\mathbb{F} R$-modules with the indecomposable $\mathbb{F S}_{p}$-modules. Repeated application of the modular Branching Theorem ([7, 9.3]) yields that $S^{\mu}$ is a direct summand of $S^{\lambda} \downarrow_{R}$ with multiplicity 1 , whence $S^{\lambda} \downarrow_{R \times G_{p}}$ has a direct summand that is the outer tensor product of $S^{\mu}$ with a 1-dimensional direct summand of $S^{\lambda} \downarrow_{\mathfrak{G}_{p}}$. It also yields that $S^{\lambda} \downarrow_{\mathfrak{G}_{p}}$ has a series of submodules whose quotients are Specht modules corresponding to partitions of $p$ different from $\left(1^{p}\right)$. We saw in the previous section that none of those Specht modules has a submodule isomorphic to $S^{\left(1^{p}\right)}$ (though one of them does have such a quotient), so we may conclude that $S^{\lambda} \downarrow_{\mathfrak{S}_{p}}$ has no 1-dimensional submodule other than $\mathbb{F}_{\mathcal{S}_{p}}$. It follows that the relevant direct summand of $S^{\lambda} \downarrow_{R \times \mathfrak{S}_{p}}$ can only be $S^{\mu} \sharp \mathbb{F}_{\mathfrak{G}_{p}}$. Of course, then $S^{\mu} \sharp \mathbb{F}_{N}$ is a (non-projective indecomposable) direct summand of $S^{\lambda} \downarrow_{R \times N}$. This proves both that $S^{\lambda}$ is non-projective and that it is the Green correspondent of $S^{\mu} \sharp \mathbb{F}_{N}$.

We have already noted that the unique $p$-hook of $\lambda$ has leg-length 0 ; equivalently, in the terminology of Nakayama [9], it has height 1 . Now we can apply [9, Theorem 4], just as it was in the proof of Theorem 1 above. First, it yields that $S^{\lambda}$ is simple. Second, it leads to the conclusions that all odd Heller translates of $S^{\lambda}$ are Specht modules or dual Specht modules corresponding to partitions of $r$ which contain $p$-hooks of odd leg-length, and that none of these modules is projective or simple. This completes the proof of Theorem 2.

\section{References}

[1] J. L. Alperin, Local representation theory, Cambridge Studies in Advanced Math. 11 (Cambridge University Press, Cambridge, 1986).

[2] N. Bourbaki, Groupes et algébres de Lie, Chap. II, III, Actualités Sci. Indust. 1349 (Hermann, Paris, 1972).

[3] A. J. Brandt, 'The free Lie ring and Lie representations of the full linear group', Trans. Amer. Math. Soc. 56 (1944), 528-536.

[4] R. M. Bryant and R. Stöhr, 'Fixed points of automorphisms of free Lie algebras', Arch. Math. 67 (1996), 281-289.

[5] _ _ 'On the module structure of free Lie algebras', Trans. Amer. Math. Soc., to appear.

[6] J. A. Green, Vorlesungen über modulare Darstellungstheorie endlicher Gruppen, Vorlesungen aus dem mathematischen Institut Giessen, Heft 2 (Manuskript: Wolfgang Hamernik) (Mathematisches Institut Giessen, Universität Giessen, Giessen, 1974).

[7] G. D. James, The representation theory of the symmetric groups, Lecture Notes in Math. 682 (Springer-Verlag, Berlin, 1978).

[8] L. G. Kovács and R. Stöhr, 'Module structure of the free Lie ring on three generators', Arch. Math. 72 (1999), to appear.

[9] T. Nakayama, 'On some modular properties of irreducible representations of symmetric groups, II', Japan. J. Math. 17 (1940), 411-423. 
[10] G. E. Wall, 'On the Lie ring of a group of prime exponent', in: Proceedings of the second international conference on the theory of groups (ed. M. F. Newman), Lecture Notes in Math. 372 (Springer-Verlag, Berlin, 1974) pp. 667-690.

[11] E. Witt, 'Die Unterringe der freien Lieschen Ringe', Math. Z. 64 (1956), 195-216.

UMIST

PO Box 88

Manchester M60 1QD

England

e-mail: bryant@umist.ac.uk

e-mail: r.stohr@umist.ac.uk
Australian National University Canberra ACT 0200

Australia

e-mail: kovacs@maths.anu.edu.au 